\title{
Vers une meilleure compréhension de l'implantation d'un programme de transfert de connaissances par agents multiplicateurs
}

\section{A train-the-trainer knowledge transfer program's evaluation for an ecological understanding of the implementation process}

\author{
Mélodie Briand-Lamarche, Julie Dutil, Luc Dargis-Damphousse et Christian \\ Dagenais
}

Volume 41, numéro 1, 2012

URI : https://id.erudit.org/iderudit/1061820ar

DOI : https://doi.org/10.7202/1061820ar

Aller au sommaire du numéro

\section{Éditeur(s)}

Revue de Psychoéducation

ISSN

1713-1782 (imprimé)

2371-6053 (numérique)

\section{Découvrir la revue}

Citer cet article

Briand-Lamarche, M., Dutil, J., Dargis-Damphousse, L. \& Dagenais, C. (2012). Vers une meilleure compréhension de l'implantation d'un programme de transfert de connaissances par agents multiplicateurs. Revue de psychoéducation, 41(1), 21-46. https://doi.org/10.7202/1061820ar

\section{Résumé de l'article}

Malgré une augmentation de l'utilisation des programmes psychosociaux basés sur des données probantes (Fraser, 2004; Gira, Kessler \& Poertner, 2004; Howard, McMillen \& Pollio, 2003), ces programmes ne sont toujours pas les plus utilisés (Ringwalt et al. 2002, Wandersman \& Florin 2003). En effet, le transfert et l'implantation des programmes sont des processus complexes et le développement d'interventions démontrées efficaces n'est qu'une étape vers l'amélioration de la santé et du bien-être des populations (Durlak \& DuPre, 2008). Le présent article a pour objectif d'expliciter le processus d'implantation du programme Agir en milieu de garde et les facteurs qui l'ont influencé. Ce programme par agents multiplicateurs mis sur pied par le Centre de liaison sur l'intervention et la prévention psychosociales (CLIPP) vise à prévenir les mauvais traitements chez les jeunes enfants en formant des agentes dans les bureaux coordonnateurs de la garde en milieu familial sur les connaissances issues de la recherche en matière de mauvais traitements envers les enfants afin que ces agentes forment à leur tour les responsables en service de garde familial de leur milieu. Après un bref retour sur les résultats du premier volet de l'étude ayant permis d'évaluer le niveau d'implantation et l'influence d'un certain nombre de facteurs organisationnels, individuels et propres au programme (Briand-Lamarche \& Dagenais, 2012), le présent article se concentre sur le second volet de l'étude. Ce second volet a permis, grâce à des entrevues semi-structurées avec 49 des agentes multiplicatrices du programme, de comprendre l'influence sur l'implantation de l'interaction des facteurs organisationnels, individuels, propres au programme et communautaires. Les résultats de ce second volet de l'étude permettent de démontrer que, comme le soulignent Durlak et DuPre (2008), une implantation efficace dépend de l'interaction de plusieurs facteurs.
Tous droits réservés @ La Corporation de la Revue Canadienne de Psycho-Éducation, 2012
Ce document est protégé par la loi sur le droit d'auteur. L’utilisation des services d’Érudit (y compris la reproduction) est assujettie à sa politique d'utilisation que vous pouvez consulter en ligne.

https://apropos.erudit.org/fr/usagers/politique-dutilisation/ 


\title{
Vers une meilleure compréhension de l'implantation d'un programme de transfert de connaissances par agents multiplicateurs
}

\author{
A train-the-trainer knowledge transfer program's evaluation \\ for an ecological understanding of the implementation \\ process
}

M. Briand-

Lamarche $^{1,2}$

J. Dutil ${ }^{2}$

L. DargisDamphousse $^{1,2}$

C. Dagenais ${ }^{1,2}$

1. Département de psychologie, Université de Montréal

2. Centre de liaison sur l'intervention et la prévention psychosociales

Correspondance :

Mélodie Briand-Lamarche 555 , boul. René-Lévesque Ouest Bureau 1200 Montréal (Québec) $\mathrm{H} 2 \mathrm{Z}$ 1B1

Tél. : 514-393-4666 poste 2019 Télecopieur : 514-393-9843 melodie.briand@gmail.com
Résumé

Malgré une augmentation de l'utilisation des programmes psychosociaux basés sur des données probantes (Fraser, 2004; Gira, Kessler \& Poertner, 2004; Howard, McMillen \& Pollio, 2003), ces programmes ne sont toujours pas les plus utilisés (Ringwalt et al. 2002, Wandersman \& Florin 2003). En effet, le transfert et l'implantation des programmes sont des processus complexes et le développement d'interventions démontrées efficaces n'est qu'une étape vers l'amélioration de la santé et du bien-être des populations (Durlak \& DuPre, 2008). Le présent article a pour objectif d'expliciter le processus d'implantation du programme Agir en milieu de garde et les facteurs qui l'ont influencé. Ce programme par agents multiplicateurs mis sur pied par le Centre de liaison sur l'intervention et la prévention psychosociales (CLIPP) vise à prévenir les mauvais traitements chez les jeunes enfants en formant des agentes dans les bureaux coordonnateurs de la garde en milieu familial sur les connaissances issues de la recherche en matière de mauvais traitements envers les enfants afin que ces agentes forment à leur tour les responsables en service de garde familial de leur milieu. Après un bref retour sur les résultats du premier volet de l'étude ayant permis d'évaluer le niveau d'implantation et l'influence d'un certain nombre de facteurs organisationnels, individuels et propres au programme (Briand-Lamarche \& Dagenais, 2012), le présent article se concentre sur le second volet de l'étude. Ce second volet a permis, grâce à des entrevues semi-structurées avec 49 des agentes multiplicatrices du programme, de comprendre l'influence sur l'implantation de l'interaction des facteurs organisationnels, individuels, propres au programme et communautaires. Les résultats de ce second volet de l'étude permettent de démontrer que, comme le soulignent Durlak et DuPre (2008), une implantation efficace dépend de l'interaction de plusieurs facteurs.

Mots-clés : Transfert de connaissances, Agents multiplicateurs, Évaluation de l'implantation. 


\begin{abstract}
Despite an increased use of psychosocial evidence-based programs (Fraser, 2004; Gira, Kessler and Poertner, 2004; Howard, McMillen and Pollio, 2003), these programs are still not the most sought after (Ringwalt et al. 2002, Wandersman and Florin 2003). Indeed, the programs transfer and implementation is a complex process and the development of interventions proven to be effective is but one step toward the improvement of general populations health and well-being (Durlak and DuPre, 2008). That is why the CLIPP wanted the implementation of its Agir en milieu de garde program to be evaluated so that it could both appreciate to what extent the program had taken root and determine what factors had influenced it. The goal of this train-the-trainer program is to prevent the abuse of young children. To do this, it trains agents in the childcare coordinating offices in matters related to research on child abuse so that these agents can, in turn, transfer that knowledge to the people in charge of family day care centers in their own community. Except for a brief review of the study's first part for the purpose of assessing the level of implementation and the influence of a several organizational, individual and program specific factors, this article focuses on the second part of the study. In this second part, a series of semi-structured interviews with 49 multiplying agents helps the reader understand how the interaction between organizational, individual, program specific and community factors has impacted the implementation. The second part of the study's results demonstrates, as noted Durlak and DuPre (2008) that for an implementation process to be effective, numerous factors must interact.
\end{abstract}

Key words: Knowledge Transfer, Train-the-Trainer Program, Program evaluation

\title{
Introduction
}

En 2006, le Centre de liaison sur l'intervention et la prévention psychosociale (CLIPP) a mis sur pied un projet de déploiement du programme de formation Agir en milieu de garde. Ce projet faisait suite à l'évaluation d'un projet-pilote d'implantation du programme réalisé sur huit sites d'expérimentation en 2004-2005 par l'organisme et ses collaborateurs. Ce programme visait à répondre aux besoins identifiés dans le cadre d'une étude effectuée en 2004 (CLIPP, 2006). Cette étude de besoin faisait état d'un désir des membres du personnel des centres de la petite enfance d'en apprendre plus sur les mauvais traitements physiques, psychologiques et sur le syndrome du bébé secoué afin d'être plus efficaces dans leurs interventions auprès des enfants et des familles se trouvant dans ces situations (CLIPP, 2004).

Étant donné la gravité et l'ampleur de la problématique des mauvais traitements physiques et psychologiques chez les enfants, il est apparu nécessaire de répondre au besoin exprimé. En effet, selon Tourigny et al. (2002), 4,5 enfants québécois sur 1000 âgés de 0 à 5 ans font l'objet d'un signalement pour abus physiques et 4,9 sur 1000 pour abus psychologiques. Toutefois, ces données ne tiennent compte que des cas connus du système de protection de la jeunesse. Selon Trocmé, McPhee, Tam et Hay (1994), les cas recensés dans les enquêtes sur la maltraitance chez les enfants pourraient bien être seulement la pointe de l'iceberg, laissant dans l'ombre les cas connus, mais non signalés ainsi que les cas inconnus; pour diverses raisons, de nombreux cas ne sont tout simplement pas signalés aux services d'aide (Delaronde, King, Bendel, \& Reece, 2000; Gaston \& Sutherland, 1999). 
Ainsi, c'est dans la perspective de sensibiliser les intervenants présents dans la vie des enfants à la problématique des mauvais traitements et à l'importance de la prévention que le CLIPP a développé le programme de formation Agir en milieu de garde. Ce programme a pour objectifs de permettre aux intervenants : 1) d'augmenter leurs connaissances de la maltraitance envers les enfants, 2) de reconnaître les signes généralement associés aux mauvais traitements, 3) d'adopter les attitudes et comportements appropriés dans ces situations et 4) d'offrir une aide précoce aux enfants affectés et à leur famille (CLIPP, 2006). Ce programme inclut une trousse comprenant du matériel didactique ainsi qu'une formation de deux jours, toutes deux basées sur les connaissances scientifiques les plus à jour. Afin de permettre la diffusion à grande échelle de ce programme dans les garderies en milieu familial, le Ministère de la Famille et des Ainés a décidé, au printemps 2007, d'assumer les coûts liés à la formation et à l'achat de trousses pour deux professionnels dans chacun des 165 bureaux coordonnateurs de la garde en milieu familial du Québec. Ce faisant, les professionnels des bureaux coordonnateurs de différentes régions du Québec ont suivi la formation entre octobre 2007 et mars 2008. Sous la responsabilité de ces bureaux coordonnateurs se trouvaient, en 2008, pour l'ensemble du Québec, 14373 responsables en service de garde certifiées. En majorité des femmes (98,5\%), elles accueillaient chacune en moyenne cette annéelà six enfants dans une structure résidentielle et selon des horaires variables. Dans l'objectif de former les agentes des bureaux coordonnateurs afin qu'elles puissent à leur tour former les responsables de leur territoire, la première journée de formation portait sur la sensibilisation à la problématique de la maltraitance chez les enfants et la seconde visait l'acquisition de techniques permettant aux agentes de mener des actions préventives auprès des responsables (CLIPP, 2008).

Le CLIPP, par souci de transparence et d'amélioration continue, a souhaité que le processus d'implantation du programme et les facteurs qui l'ont influencé soient évalués. La présente étude vise donc à comprendre comment le programme a été implanté par les agents multiplicateurs formés par le CLIPP et en quoi les caractéristiques du programme, celles des agents multiplicateurs, de leur organisation et de leur communauté ont eu un impact sur cette implantation.

\section{Contexte théorique}

Bien que, dans les dernières années, une recrudescence de l'intérêt porté à l'utilisation de programmes fondés sur des données probantes en sciences sociales a été constatée (Fraser, 2004; Gira, Kessler, \& Poertner, 2004; Howard et al., 2003), il n'en demeure pas moins que ce type d'innovation n'est pas toujours le plus utilisé (Ringwalt et al., 2002; Wandersman \& Florin 2003). Un des moyens permettant de réduire le fossé entre recherche et pratique afin de mettre de l'avant les programmes basés sur des données probantes est de miser sur le savoir des milieux de pratique lors de l'implantation de ces programmes (Lee, Altschul \& Mowbray, 2008). C'est la stratégie qu'a adoptée le CLIPP pour le programme Agir en milieu de garde, et ce, en optant pour une implantation par agents multiplicateurs. Les programmes de formation par agents multiplicateurs découlent d'un modèle éducationnel grâce auquel une institution détenant une expertise sur un sujet donné transmet ce savoir par l'intermédiaire des formateurs qu'elle choisit à même le milieu visé par les formations. Ces formateurs reçoivent une formation, du matériel 
éducatif et des instructions quant à l'implantation qui leur permettent de jouer ce rôle dans leur communauté (Orfaly et al., 2005). En misant sur des formateurs issus du milieu visé, les programmes de formation par agents multiplicateurs profitent de la crédibilité de ces derniers et du lien de confiance établi avec leur milieu (Orfaly et al., 2005). Ce faisant, ces programmes bénéficient non seulement de la crédibilité scientifique de l'institution qui les initie, mais aussi du fait que l'information soit transmise à la communauté par des formateurs en qui celle-ci a confiance (McClelland, Irving, Mitchell, Bearon et Webber, 2002). Levy, Hirsch, Aghababian, Segall et Vanderschmidt (1999) ont démontré que les apprentissages effectués par les participants lors de formations par agents multiplicateurs sont équivalents aux apprentissages faits par ces derniers lors d'une formation initiale. Hinds, Patterson et Pfeffer (2001) expliquent que les agents multiplicateurs pourraient même être de meilleurs agents de transfert de connaissances que des formateurs experts, parce qu'ils sont plus à même d'utiliser un langage clair et d'incorporer à leurs formations des exemples concrets. Toutefois, les formations par agents multiplicateurs ont deux inconvénients majeurs au niveau de l'implantation. D'une part, moins de la moitié des agents multiplicateurs formés jouent ensuite leur rôle de formateurs. D'autre part, bien qu'ils transmettent correctement le contenu qu'ils choisissent d'implanter, les agents multiplicateurs ne sont pas toujours fidèles à l'ensemble du contenu, négligeant parfois d'implanter certains éléments, notamment ceux considérés comme plus innovants (Hahn, Noland, Rayens \& Christie, 2002).

Toutefois, ces embûches au niveau de l'implantation ne seraient pas uniques aux programmes de formation par agents multiplicateurs. Selon Durlak et DuPre (2008), l'implantation de programme parfaitement conforme à ce qui est prévu est peu probable. En effet, les résultats d'une méta-analyse de plus de 500 études sur le sujet montrent qu'un niveau d'implantation de $60 \%$ est jugé positif, que peu d'études rapportent un niveau d'implantation au-delà de $80 \%$ et qu'aucune ne documente une implantation de 100 \% (Durlak \& DuPre, 2008). Le transfert de connaissances et l'implantation des programmes sont des processus complexes. En ce sens, le développement d'interventions démontrées efficaces n'est qu'une étape vers l'amélioration de la santé et du bien-être des populations (Durlak \& DuPre, 2008). Afin d'améliorer le processus de transfert de connaissances visant à réduire le fossé entre la recherche et la pratique, la compréhension de la capacité des acteurs de l'implantation, c'est-à-dire de leur motivation et de leurs compétences, est cruciale (Goodman et al., 1998; Miller \& Shinn, 2005; Schorr, 2003; Wandersman, 2003). Selon Wandersman et al. (2008), la capacité des acteurs de l'implantation influence cette dernière via trois catégories de facteurs : les facteurs individuels (par exemple le sentiment d'efficacité personnelle), organisationnels (par exemple les ressources telles que le temps ou le soutien) et communautaires (par exemple le niveau de défavorisation). Greenhalgh, Robert, MacFarlane, Bate et Kyriakidou (2004) soulignent pour leur part que les facteurs organisationnels interagissent non seulement entre eux, mais aussi avec les caractéristiques du programme à implanter. 


\section{Objectifs et contexte de l'étude}

Le premier volet de la présente étude visait à documenter l'implantation du programme Agir en milieu de garde (Briand-Lamarche \& Dagenais, 2012). Les facteurs organisationnels, individuels et propres au programme ont aussi, dans le cadre de ce premier volet, été examinés grâce à une méthodologie quantitative afin de déterminer jusqu'à quel point ils influencent le niveau d'implantation. Les résultats de ce volet démontrent que près de la moitié des agentes multiplicatrices $(46,4 \%)$ ont tenu des activités de transfert auprès des responsables en service de garde de leur territoire. La mesure de la fidélité d'implantation, représentant le niveau avec lequel le contenu des activités de transfert offertes correspond au contenu du programme (Dane \& Schneider, 1998), est de 65,19\% en moyenne pour les 58 agentes multiplicatrices ayant offert des activités de transfert. La fidélité moyenne varie toutefois selon les thématiques, allant de $53,38 \%$ à $81,04 \%$.

L'étude de facteurs organisationnels, individuels et propres au programme dans ce premier volet de l'étude permet de constater que c'est le temps dont disposent les agentes multiplicatrices pour préparer et animer des activités de transfert qui est le plus fortement corrélé avec la fidélité d'implantation $(r=0,50$, $p<.01)$. Le sentiment d'efficacité personnelle est aussi en corrélation avec la fidélité d'implantation $(r=0,33, p<.01)$, de même que la perception que le programme répond à un besoin $(r=0,22, p<.05)$.

Au cours de ce premier volet, les influences des facteurs organisationnels, individuels et propres au programme ont été étudiées individuellement afin de déterminer l'influence de chacun de ces facteurs sur le processus d'implantation du programme Agir en milieu de garde. Cela dit, tel que mentionné précédemment, selon Durlak et DuPre (2008), le succès d'une implantation dépend non seulement des facteurs organisationnels, individuels et propres au programme ainsi que des caractéristiques de la communauté dans laquelle est implanté le programme, mais aussi des interactions entre ces quatre catégories de facteurs. Ainsi, une vision écologique de ces différents facteurs permet de mieux comprendre le processus d'implantation d'un programme (Altschuld, Kumar, Smith \& Goodway, 1999; Durlak \& DuPre, 2008; Riley, Taylor, \& Elliott, 2001; Shediac-Rizkallah \& Bone, 1998; Wandersman, 2003). C'est pourquoi le second volet de l'étude décrit dans cet article étudie, grâce à une méthodologie qualitative exploratoire, ce qui a influencé l'implantation d'activités de transfert par les agentes multiplicatrices du programme Agir en milieu de garde. Ce volet vise à donner la parole aux agentes afin qu'elles expriment ce qui a influencé le processus d'implantation au-delà du cadre restrictif d'un questionnaire à choix de réponses tel que celui utilisé dans le premier volet de l'étude. II est attendu que cela permettra de contribuer à l'obtention d'une compréhension écologiquement valide du processus d'implantation du programme Agir en milieu de garde. L'objectif de ce volet est de comprendre comment les caractéristiques du programme, des agentes multiplicatrices, du milieu organisationnel (les bureaux coordonnateurs) et de la communauté ainsi que leurs interactions ont influencé le processus d'implantation du programme Agir en milieu de garde. En accord avec les postulats ontologiques de la recherche qualitative qui permettent un processus de recherche itératif où les questions, les hypothèses et les variables ne sont pas complètements prédéterminés (Lessard-Hébert, Goyette, 
\& Boutin, 1996), le présent volet de l'évaluation est particulièrement sensible à la réalité des agentes multiplicatrices dans le cadre de l'implantation du programme Agir en milieu de garde. Cette sensibilité à la réalité des agentes que permet la méthode qualitative fait en sorte que le présent volet de l'évaluation étudie l'implantation et les facteurs qui l'ont influencé sans en dénaturer le contexte, en accord avec la définition que donne Bronfenbrenner (1977) de la validité écologique comme étant le niveau avec lequel l'expérience vécue par le sujet d'une expérimentation est celle que suppose le chercheur.

\section{Méthode}

Tel que mentionné précédemment, Durlak et DuPre (2008) soulignent que ce qui est requis pour une implantation efficace est propre à chaque contexte. Par ailleurs, Strauss et Corbin (1990) expliquent que l'utilisation de méthodes qualitatives est appropriée afin de mieux comprendre un phénomène pour lequel peu de connaissances sont disponibles ou pour mieux saisir des détails difficilement mesurables à l'aide d'une méthodologie quantitative. II importe de souligner que cette étude a été menée à bien avec l'autorisation du Comité d'éthique de la recherche de la Faculté des arts et des sciences de l'Université de Montréal.

\section{Participantes}

Parmi les 370 agentes multiplicatrices formées par le CLIPP, 55 ont été retenues pour participer à des entretiens, parmi lesquelles 49 ont effectivement accepté de participer à un entretien. Parmi elles, 26 avaient effectué des activités au moment de la collecte de données. Une méthode d'échantillonnage aléatoire stratifiée a été privilégiée pour favoriser la représentativité de l'échantillon et pour réduire les biais d'échantillonnage (Fernandes, Kamienski, Kelner, Mariz, \& Sadok, 2008; Poupart, Deslauriers, Groulx, Laperrière, Mayer, \& Pires, 1997; Tashakkori \& Teddlie, 2003; Van Der Maren, 1996). Plus spécifiquement, les participantes à l'évaluation ont été sélectionnées de manière aléatoire, à partir d'une base de données Excel, parmi toutes les agentes multiplicatrices formées par le CLIPP. L'échantillonnage a été stratifié en fonction des régions administratives du Québec. En effet, afin de s'assurer que ce volet de l'étude couvrait toutes les régions où des agentes ont été formées, une sélection aléatoire a été réalisée dans certaines d'entre elles.

Étant donné la stratification effectuée, les participantes proviennent des 17 régions administratives du Québec. Comme le résume le Tableau 1, elles occupent en majorité un poste d'agente pédagogique, ont entre moins d'un an et 30 ans d'expérience dans leur emploi et détiennent toutes un diplôme collégial ou universitaire. 
Tableau 1 : Renseignements sociodémographiques des participantes

\begin{tabular}{lcc}
\hline & $\begin{array}{c}\text { Ensemble des agentes } \\
\text { formées }(\mathrm{N}=370)\end{array}$ & Échantillon $(\mathrm{n}=49)$ \\
\hline $\begin{array}{l}\text { Expérience dans leur } \\
\text { domaine d'emploi }\end{array}$ & & \\
$\quad$ Moins d'un an & 13,6 & 9,9 \\
$\quad$ Entre 1 an et $<2$ ans & 35,2 & 33,3 \\
$\quad$ Entre 2 ans et $<3$ ans & 8,7 & 11,9 \\
$\quad$ Entre 3 et 30 ans & 42,5 & 45,2 \\
Dernier diplôme obtenu & & \\
$\quad$ Formation universitaire & 54,5 & 56,1 \\
$\quad$ Formation collégiale & 45,5 & 43,9 \\
Poste occupé & & \\
$\quad$ Soutien pédagogique & 50,0 & 59,2 \\
$\quad$ Agente de conformité & 28,9 & 12,2 \\
$\quad$ Autres & 21,1 & 28,6 \\
\hline
\end{tabular}

\section{Instrument de mesure}

Des entretiens semi-structurés d'une durée de 30 à 60 minutes avec les agentes multiplicatrices ont été réalisés. Ce type d'entretien permet d'aborder systématiquement les mêmes thèmes avec chaque participante, mais la séquence et le libellé des questions peuvent être adaptés à chaque situation. Il permet également d'approfondir les thèmes qui ressortent des entretiens (Lessard-Hébert, Goyette, \& Boutin, 1996; Patton, 1990). Afin de cerner les choix d'implantation et le processus d'influence de ces choix, plusieurs thèmes ont été abordés lors des entretiens. Pour celles qui, au moment de la collecte des données, avaient déjà eu l'occasion de réaliser des activités de transfert ${ }^{1}(n=26)$, les questions d'entrevue portaient sur les activités implantées et sur les conditions susceptibles de faciliter ou de nuire à l'implantation de ces activités. Les thèmes abordés étaient : le contenu des activités et les méthodes pédagogiques de présentation, le contexte organisationnel (horaire et disponibilité des collègues de travail, stabilité du personnel, collaboration de l'employeur, matériel de présentation disponible dans le milieu, etc.) et leur vision personnelle du programme (déroulement de la préparation et de la présentation de l'activité, satisfaction par rapport à la trousse et à la formation offerte par le CLIPP, etc.). Pour les agentes multiplicatrices qui n'avaient pas encore eu l'occasion de réaliser des activités de transfert $(n=23)$, les dimensions retenues portaient sur la reconnaissance du rôle d'agente multiplicatrice par leur milieu organisationnel, les motifs expliquant le report des activités et les prévisions quant à la tenue éventuelle de celles-ci.

1. Le terme " activités de transfert » est utilisé lorsqu'il est question indifféremment d'activités de formation ou d'activités d'information. 


\section{Procédures d'administration}

Les entretiens avec les agentes multiplicatrices ont été effectués par téléphone. À l'aide de l'obtention du consentement verbal, des rendez-vous téléphoniques ont été pris avec les participantes afin de réaliser les entretiens environ un mois après la date prévue pour la tenue de leurs activités de transfert. L'information concernant la date prévue des activités qu'elles offriraient avait été obtenue préalablement lors de contacts téléphoniques avec elles dans les mois suivant la formation du CLIPP. Avec l'autorisation des participantes, les entrevues ont, par la suite, été enregistrées et retranscrites intégralement.

\section{Procédures d'analyses}

Les procédures d'analyse des entrevues s'inspirent de la méthode proposée par Miles et Huberman (2003). Elles se déroulent en trois phases. Tout d'abord, les entrevues sont condensées, c'est-à-dire qu'elles sont découpées en fonction de codes correspondant aux thèmes abordés lors des entrevues. Ces codes peuvent être appliqués à un mot, à une phrase ou à un paragraphe en entier. Cette phase permet d'organiser les propos des répondantes en fonction des idées et des thèmes qu'ils contiennent de manière à pouvoir réaliser des opérations analytiques (Lessard-Hébert et al., 1996).

La seconde phase consiste à présenter et à organiser les données. Lors de cette phase, des opérations analytiques sont réalisées à partir de codes appliqués dans la phase précédente. Cela permet d'organiser les discours des répondantes afin de pouvoir les interpréter. Dans le cadre de la présente étude, les discours ont donc été regroupés selon les facteurs d'influence (individuels, organisationnels, communautaires, propres à l'innovation). La dernière phase d'analyse des données consiste à les interpréter et à les vérifier. II s'agit de produire des connaissances à partir de l'organisation des discours (Miles \& Huberman, 2003). C'est lors de cette dernière phase qu'ont été mis en lien les propos des participantes quant à leurs activités de transfert ainsi que leurs propos quant aux différents facteurs d'influence afin de formuler des inférences sur la manière dont les facteurs ont influencé le processus d'implantation des activités de transfert.

Les analyses ont été effectuées par une équipe de trois chercheurs afin d'assurer un maximum de validité interne aux résultats qui en sont issus. En effet, le travail d'équipe lors de l'analyse des données permet une meilleure prise en compte du positionnement de chaque chercheur au plan théorique et épistémologique afin de mieux se distancer de ces positions par la suite et en limiter l'influence sur les résultats émergents (Poupart et al., 1997). 


\section{Résultats}

\section{Constats préliminaires}

Le premier constat qui s'impose grâce à l'analyse des entretiens avec les agentes multiplicatrices est qu'une grande part du processus d'implantation du programme s'effectue autour d'une première grande décision, soit le choix du type d'activités à implanter. À ce niveau, les entretiens ont permis de constater que les agentes multiplicatrices évaluent la possibilité d'implanter l'un ou l'autre de deux grands types d'activités : des activités de formation ou d'informations. La définition de ces deux types d'activités varie d'une agente multiplicatrice et d'un bureau coordonnateur à un autre, mais un certain nombre d'éléments permettent de distinguer les activités d'information et les activités de formation. C'est sur cette première décision d'implantation que s'exerce en grande partie le jeu des différents facteurs d'influence (individuels, organisationnels, communautaires et propres à l'innovation) et le type d'activité choisi semble avoir des conséquences sur le niveau d'implantation.

Les responsables en service de garde ont l'obligation de suivre six heures d'activités de formation annuellement. Les bureaux coordonnateurs offrent à leurs responsables un certain nombre de formations dans lesquelles elles doivent faire un choix afin de remplir cette obligation. La participation à une activité de formation n'est donc pas entièrement volontaire puisque les responsables ont l'obligation de suivre six heures de formation, mais pour ce faire, elles ont le choix des activités auxquelles elles participent, parmi les activités sélectionnées par le bureau coordonnateur. Pour bénéficier du titre de formation et faire partie du choix annuel des responsables en service de garde, le contenu d'une activité doit être directement en lien avec la tâche quotidienne de celles-ci. Par ailleurs, les outils et les connaissances présentés dans le cadre d'une activité de formation doivent provenir d'une source jugée crédible par la direction du bureau coordonnateur. Les connaissances et les outils présentés dans ces activités sont transmis à titre formel et les activités de formation prennent souvent la forme de présentations magistrales

Pour leur part, les activités d'information sont des activités où la présence des responsables en service de garde est entièrement volontaire. Une activité d'information peut porter sur tous sujets jugés pertinents par le bureau coordonnateur. Les outils et les connaissances présentés dans les activités d'information peuvent provenir de différentes sources, selon les connaissances et le bon jugement des agentes de soutien qui offrent ces activités. Les activités d'information sont des lieux d'échange et de discussion entre agentes et responsables plutôt que des activités formelles d'enseignement.

Par ailleurs, les facteurs qui ont influencé les agentes multiplicatrices dans le choix d'implanter ou non le programme Agir en milieu de garde et du type d'activité pour ce faire se regroupent autour de trois axes principaux : 1) la perception qu'elles ont des besoins en formation dans leur milieu, 2) leur sentiment d'efficacité personnelle et 3 ) le temps disponible pour préparer et offrir des activités de formation ou d'information. Le rôle joué dans le processus d'implantation par 
les différents niveaux d'influence (individuels, organisationnels, communautaires et propres à l'innovation) ainsi que leurs interactions est analysé et présenté dans les sections suivantes en fonction de ces trois axes.

\section{Axe 1 : Influence de la perception des besoins}

Selon les agentes multiplicatrices, le sujet abordé par le programme de formation Agir en milieu de garde, les mauvais traitements envers les jeunes enfants, est fort important. Une agente mentionne :

"Je pense, moi, que ce qui a été le plus intéressant, c'est le contenu. Le sujet est intéressant. Le sujet est quelque chose qui va chercher nos cordes sensibles en tant qu'éducatrices, que mères, que parents, que responsables en service de garde, qu'intervenantes auprès des enfants. C'est un sujet qui nous préoccupe énormément. Alors, je pense que le sujet, le contenu, fait toute la différence dans cette formation-là. Ce serait une formation sur l'alimentation, ça n'aurait pas le même impact que ce sujet-là. »

Toutefois, en ce qui concerne le contenu du programme et la réponse qu'il pourrait offrir à des besoins spécifiques des responsables en service de garde, les réponses sont plus diversifiées et plus nuancées. Ces réponses sont influencées aussi bien par les caractéristiques du programme que par celles des agentes et des milieux organisationnels et communautaires dans lesquels elles travaillent.

Rôle des caractéristiques du programme.

Les agentes multiplicatrices interrogées ont des perceptions diverses des besoins auxquels répond le contenu du programme. Pour certaines, les activités de transfert permettront aux responsables en service de garde de mieux prévenir et détecter des situations de mauvais traitements subis par les enfants dans leur milieu familial. D'autres sont d'avis que ces activités rendent les responsables en service de garde plus conscientes de leurs propres agissements, faisant en sorte qu'elles pourront mieux prévenir d'éventuels mauvais traitements envers les enfants dont elles seraient elles-mêmes les instigatrices. Les agentes multiplicatrices expliquent aussi que le fait que ce soit elles qui offrent les activités de transfert aux responsables renforce le lien de confiance qu'ont ces dernières envers elles. Ainsi, les responsables réalisent que les agentes de leur bureau coordonnateur sont là pour les aider lorsque des situations potentielles de mauvais traitements se présentent. Ce faisant, les répondantes pensent que les responsables en service de garde se sentiront plus à l'aise de discuter d'éventuelles situations de mauvais traitements dans leur service de garde, un sujet qu'elles hésitent souvent à aborder avec les agentes.

Les agentes mentionnent que le contenu du programme faisant référence à des situations concrètes, telles que les études de cas suggérées dans la trousse, les DVD et l'énonciation des facteurs de risques et de protection sont particulièrement pertinents pour répondre à ces besoins, c'est-à-dire pour sensibiliser les responsables et pour ouvrir des discussions pouvant éventuellement mener à un 
meilleur lien de confiance. Quelques limites ont aussi été mentionnées. Certaines agentes ont exprimé qu'un plus grand nombre d'exemples concrets et d'études de cas aurait été utile. Aussi, le temps nécessaire lors des activités de transfert pour entrer en interaction avec les responsables en services de garde, pour discuter des études de cas, pour répondre aux questions et aux interventions des participantes a été, selon les agentes, largement sous-estimé dans les suggestions d'implantation incluses dans la trousse. Cela fait en sorte que les agentes ont senti la nécessité de choisir entre investir plus de temps dans l'explication du contenu théorique ou dans les discussions et interactions avec les participantes puisqu'elles n'avaient pas prévu un temps de formation suffisant pour accomplir ces deux tâches.

\section{Rôle des caractéristiques individuelles des agentes multiplicatrices.}

Bien que le sujet des mauvais traitements est un thème important, les agentes multiplicatrices interrogées ont mentionné que nombre d'autres sujets doivent être abordés auprès des responsables en service de garde. Les moyens d'évaluer les besoins en formation varient d'un bureau coordonnateur à l'autre, mais il revient souvent aux agentes de faire des recommandations à ce propos et il appert que le vécu de ces agentes en matière de mauvais traitements pourrait influencer la priorisation de ce sujet dans les besoins de formation. Certaines agentes multiplicatrices ont exprimé qu'elles constatent, dans le quotidien des responsables en services de garde, des besoins de formation plus pressants que la sensibilisation aux mauvais traitements. D'autres agentes expliquent toutefois que bien que les mauvais traitements ne soient pas un problème quotidien, celui-ci doit être traité de manière prioritaire. Ces dernières justifient souvent ce point de vue par une expérience préalable de sensibilisation aux mauvais traitements. En d'autres termes, les agentes ayant soit vécu des expériences liées à des situations de mauvais traitements ou ayant suivi précédemment des formations sur le sujet semblent être plus sensibles et plus portées à considérer le thème des mauvais traitements prioritaire.

\section{Rôle des caractéristiques organisationnelles.}

Puisque les bureaux coordonnateurs sont situés dans des milieux forts différents les uns des autres, les caractéristiques de ces milieux ont une influence non négligeable sur les besoins des responsables en service de garde qui y travaillent. Certaines agentes multiplicatrices ont mentionné que le fait que leur bureau coordonnateur soit situé en milieu défavorisé a influencé leur perception des besoins des responsables en service de garde en matière de formation, les encourageant à offrir la formation Agir en milieu de garde. Il est en effet expliqué dans le contenu du programme que la défavorisation du milieu représente un facteur de risque de maltraitance (CLIPP, 2006).

Rôle de la communauté.

Un certain nombre de bureaux coordonnateurs situés dans des milieux plus défavorisés font partie d'un projet intitulé Grandir ensemble. Ce projet vise à : " développer et consolider l'accueil et la rétention des enfants issus de milieux défavorisés ", et ce, à travers des activités de sensibilisation des différents 
acteurs des milieux de garde aux divers enjeux de l'intervention en contexte de défavorisation (Fondation Lucie et André Chagnon, 2009).

Certaines des agentes multiplicatrices prenant part à ce projet mentionnent avoir pu assister à des activités de formations portant sur les sujets de mauvais traitements ou des sujets connexes liés à la défavorisation. Cela a pu influencer leur perception de la problématique et l'évaluation qu'elles font du besoin de formation des responsables en service de garde de leur territoire, tel que mentionné précédemment. Cette communauté d'apprentissage a aussi aidé les agentes multiplicatrices qui y participent à mettre en commun des exemples concrets et des études de cas créées en supplément de celles fournies dans la trousse de formation, de manière à répondre adéquatement aux besoins perçus chez leurs responsables en service de garde malgré les limites du programme.

Par ailleurs, les agentes multiplicatrices ayant des contacts fréquents avec les différents organismes et instances de leur communauté (CSSS, Centres jeunesse, etc.) ont mentionné avoir eu de la facilité à trouver des présentateurs pouvant pallier aux limites du programme, entre autres en venant parler aux responsables en service de garde des différentes dispositions légales dans les situations de mauvais traitements.

Résumé des influences et conséquences sur l'implantation.

Les agentes multiplicatrices sont conscientes que la problématique des mauvais traitements chez les jeunes enfants est un sujet important qui doit être abordé avec les responsables en service de garde. Toutefois, la priorité accordée à ce sujet par rapport à d'autres thématiques de formation est sujette à l'influence de plusieurs facteurs. D'une part, la présence d'éléments concrets dans la trousse de formation, tels que les études de cas et le contenu des DVD, fait en sorte que les agentes pensent être à même d'offrir des activités de transfert aux responsables de manière à bien pouvoir répondre aux besoins cernés, malgré certaines limites. La manière dont ces besoins sont cernés dépend par ailleurs, au-delà du contenu du programme, des expériences personnelles des agentes et des caractéristiques socio-économiques du milieu dans lequel elles travaillent. Le lien qu'entretiennent les agentes avec ce milieu, ses instances et ses organismes ainsi que les contacts qu'elles ont avec d'autres agentes travaillant dans des milieux similaires influencent aussi le choix qu'elles font quant au type d'activité qu'elles implantent dans le cadre du programme Agir en milieu de garde. Les agentes ont exprimé, en entrevue, la préoccupation de rejoindre, par leurs activités de transfert, un maximum de responsables en service de garde étant donné l'importance qu'elles accordent au problème des mauvais traitements. Toutefois, le choix du type d'activités (formation ou information) ainsi que leur implantation ne dépend pas seulement de la perception des agentes des besoins en formation de leurs responsables, mais aussi de leur sentiment d'efficacité personnelle à offrir ces activités et du temps disponible dans leur horaire pour ce faire. 


\section{Axe 2 : Influence du sentiment d'efficacité personnelle des agentes multiplicatrices}

En vue de mener ou non à bien des activités auprès des responsables en service de garde et de leur donner la forme d'activités de formation ou d'information, les agentes multiplicatrices ont insisté sur l'importance de l'influence de leur sentiment d'efficacité personnelle. N'ayant pas toujours été sélectionnées par leur bureau coordonnateur dans la perspective qu'elles deviennent des agentes multiplicatrices et n'ayant pas non plus toujours été avisées de cet objectif du programme, les participantes n'avaient pas toutes la même confiance en leur capacité à jouer ce rôle. Toutefois, au-delà du fait qu'elles aient été ou non prévenues et prêtes à jouer le rôle d'agente multiplicatrice au départ, les caractéristiques du programme, les caractéristiques personnelles des agentes ainsi que les caractéristiques organisationnelles et communautaires ont contribué à nourrir ou à miner le développement du sentiment d'efficacité personnelle des agentes.

Rôle des caractéristiques du programme.

Parce que les agentes multiplicatrices jugent la thématique des mauvais traitements chez les jeunes enfants comme importante, elles ont exprimé en entrevues qu'elles ressentaient une pression à transmettre le contenu du programme de la manière la plus exacte et appropriée possible. Une agente explique :

« ... j'ai trouvé ça très intéressant et je vois l'importance de l'offrir aux responsables, mais il y a une bonne préparation à y avoir. Je ne veux pas offrir ça comme ça et pas vraiment préparée, parce que je trouve que c'est important. Et c'est de voir aussi... aux personnes à qui je vais le présenter. J'essaie de penser qu'il peut arriver... qu'il y a peut-être des personnes làdedans qui ont déjà subi des choses comme ça et je veux... il faut que je sois préparée à ça. »

La peur d'induire les responsables en service de garde en erreur ou de semer la confusion face à un problème important à leurs yeux semble avoir introduit un doute dans l'esprit d'un certain nombre d'agentes quant à leur capacité de transmettre le contenu du programme. De plus, ayant été informées lors de la formation offerte par le CLIPP que le contenu du programme pouvait créer, chez certaines responsables en service de garde, des réactions émotives, des doutes ou des questions quant aux comportements à adopter en situation de mauvais traitements, les agentes multiplicatrices envisageaient avec appréhension ces aspects imprévisibles du déroulement des activités.

Cela dit, les agentes ont vu leur sentiment d'efficacité personnelle augmenter grâce au fait que le programme offre une formation "clé en main ». Le contenu de la formation ayant déjà fait l'objet de recherche et de vérification, cela donnait crédibilité et assurance aux agentes et aux propos qu'elles tiennent lors de leurs activités. Une agente explique : 
« ... c'est le fait aussi qu'on sentait que c'était appuyé sur des recherches qui avaient été faites. Ce n'était pas des informations... C'est ça que je vous dis, on se sentait très à l'aise de livrer la marchandise, parce qu'on faisait confiance. Parce que des fois, bon, c'est sûr qu'on va donner des informations, on se dit : "Voyons, c'est-tu vrai ça, c'est-tu vérifié?" Mais là, on se sentait à l'aise, c'était appuyé. »

Aussi le fait qu'un minimum de deux agentes par bureau coordonnateur, dans la plupart des bureaux, ont été formées pour devenir des agentes multiplicatrices fait en sorte que celles-ci ont pu travailler en équipe afin de préparer et de présenter leurs activités. Les agentes ont expliqué que cela leur apporte une certaine confiance puisqu'elles peuvent diviser le contenu de manière à présenter les parties avec lesquelles elles sont le plus à l'aise. Être deux pour présenter les activités comporte aussi l'avantage de pouvoir compter sur une collègue en cas d'imprévus tels que des réactions fortes des responsables où un bris du matériel de présentation (lecteur DVD, téléviseur, projecteur, etc.).

Le fait que les agentes multiplicatrices aient participé à la formation offerte par le CLIPP leur a par ailleurs permis, selon elles, de découvrir le contenu du programme et sa trousse plus aisément que si elles n'avaient eu en leur possession que du matériel didactique. La formation leur a aussi offert un exemple d'animation faisant en sorte qu'elles se sentaient plus compétentes pour reproduire cet exemple que si elles n'avaient bénéficié que de matériel didactique sans formation initiale. Une agente mentionne :

" Enfin, moi je pense que la formation, pour moi, offerte par le CLIPP de deux jours a été nécessaire parce que ça a suscité l'intérêt, première des choses. Parce que je l'ai fait, je pouvais me référer à ce que j'avais fait lors de la formation pour pouvoir la donner. Je la comprenais davantage. "

Rôle des caractéristiques individuelles des agentes multiplicatrices.

Certaines agentes multiplicatrices ont mentionné que leur timidité à s'exprimer devant leurs collègues rendait ardue la tâche d'animer des activités de transfert. De plus, un plus haut niveau d'expérience en animation a fait en sorte que cela a paru plus aisé pour certaines agentes multiplicatrices que pour d'autres. L'influence de ces caractéristiques personnelles a toutefois rarement été exprimée sans la mention d'autres facteurs plus influents. Le plus souvent, les agentes multiplicatrices relativisaient l'influence de leur niveau d'expérience ou de timidité pour mettre de l'avant le fait que la thématique des mauvais traitements leur semble particulièrement difficile à aborder pour les raisons mentionnées précédemment. Aussi, bien que cette difficulté ait paru unanime, le niveau de sensibilité des agentes face à la thématique est une variable individuelle qui a influencé davantage le sentiment d'efficacité personnelle de certaines agentes.

Rôle des caractéristiques organisationnelles.

Des nuances existent dans les différents bureaux coordonnateurs quant au mandat des agentes en matière d'activités de formation. Dans certains bureaux, 
les agentes ont aussi bien le mandat d'organiser que d'animer des activités de transfert pour les responsables en service de garde, qu'il s'agisse d'activités de formation ou d'activités d'information. Dans d'autres bureaux, l'animation d'activités de formation revêt un caractère formel et est réservée à des formatrices externes dont le bureau coordonnateur reconnaît la compétence. Dans d'autres bureaux encore, le mandat d'animer des activités de formation est partagé entre les agentes du bureau coordonnateur et des formatrices externes. Ces différences dans le mode de fonctionnement des bureaux coordonnateurs ont un impact sur le niveau d'expérience qu'ont les agentes en animation d'activité de formation, ce qui influence leur sentiment d'efficacité personnelle tel que mentionné précédemment. De plus, dans les bureaux coordonnateurs où des formatrices externes sont préférées pour animer les activités de formation, les agentes multiplicatrices ont exprimé qu'elles sentent que leur organisation ne leur accorde pas la crédibilité ou la compétence nécessaire pour jouer le rôle de formatrice auprès des responsables en service de garde. Dans certains bureaux, cela s'est soldé par l'absence d'activités de transfert alors que dans d'autres bureaux, cela a justifié le choix de présenter des activités d'information plutôt que de formation. Dans certains bureaux, les agentes ont pu, étant donné le caractère "clé en main " du programme et le fait que cela offre une certaine garantie sur la qualité du contenu, obtenir l'autorisation exceptionnelle d'animer des activités de formation bien que cela ne fasse pas partie de leur mandat habituel. Toutefois, même lorsque les agentes ont obtenu cette autorisation, la mise en doute, dans le cadre de leur mandat habituel, de leur crédibilité ou de leur compétence en tant que formatrice a affecté leur sentiment d'efficacité personnelle en tant qu'agentes multiplicatrices.

Rôle de la communauté.

Les agentes multiplicatrices qui font partie du projet Grandir ensemble ont bénéficié d'un lieu où les échanges sur leurs expériences de présentation d'activités de transfert dans le cadre du programme ont contribué à renforcer leur sentiment d'efficacité personnelle. Puisque les agentes multiplicatrices n'étaient pas tenues, dans le cadre du programme, d'offrir leurs activités à un moment précis, certaines agentes ont procédé avant d'autres, permettant ainsi d'enrichir la préparation de ces dernières face aux éléments les plus imprévisibles.

La participation à ce projet a aussi permis à certaines agentes multiplicatrices d'assister à des formations sur des thématiques connexes à celle des mauvais traitements envers les jeunes enfants (par exemple les dispositions de la Loi sur la protection de la jeunesse). Cela a renforcé d'autant l'impression qu'elles ont d'être adéquatement outillées pour répondre aux éventuelles questions des responsables en service de garde.

\section{Résumé des influences et conséquences sur l'implantation.}

Que ce soit le risque des réactions imprévisibles des responsables face à la thématique, la timidité ou la mise en doute de la compétence et/ou de la crédibilité des agentes à agir comme formatrice, les menaces au sentiment d'efficacité personnelle des agentes multiplicatrices ont été largement nommées par ces dernières. La solution adoptée par nombre d'entre elles face à ces doutes quant à 


\section{6}

leurs capacités est de présenter le contenu sous forme d'information plutôt que de formation. Ce faisant, les agentes multiplicatrices ont agi à titre d'accompagnatrices informelles face au contenu présenté plutôt qu'à titre de formatrices.

La formule même des activités d'information, encourageant les responsables en service de garde à discuter et à échanger sur le matériel présenté plutôt que d'assister à une présentation magistrale, a rendu certaines parties du contenu plus longues à présenter que prévu. Ainsi, la présentation sous forme d'activité d'information plutôt que de formation a parfois occasionné des modifications dans le contenu présenté puisque les agentes ont dû abandonner la présentation de certaines informations au profit des échanges avec les participantes. Parfois, prévoyant ce temps nécessaire aux échanges, les agentes ont aussi dû réduire le nombre de participantes assistant aux activités d'information de manière à limiter la portée des discussions et à s'assurer d'avoir le temps nécessaire pour présenter l'ensemble du contenu prévu.

\section{Axe 3 : Influence du temps disponible}

La nécessité pour les agentes de faire des choix en matière de thématiques de formation afin de prioriser les besoins les plus pressants ainsi que leur capacité à présenter le contenu du programme de la manière la plus exacte soulignent la centralité de l'influence du temps disponible dans les choix d'implantation d'Agir en milieu de garde. Il en va de même du sentiment d'efficacité personnelle des agentes, c'est-à-dire de leurs inquiétudes quant à leur capacité à présenter adéquatement le contenu, qui est directement lié à leur besoin de prendre le temps nécessaire pour préparer leurs activités.

\section{Rôle des caractéristiques du programme.}

Le fait que le programme offre une formation "clé en main " a contribué à diminuer l'impact du manque de temps disponible chez les agentes sur le niveau d'implantation du programme. Puisque le contenu des activités de transfert était offert aux agentes par la trousse de formation, ces dernières ont affirmé qu'elles n'ont pas eu à consacrer beaucoup de temps à des recherches supplémentaires et qu'elles ont pu présenter le contenu tel quel lors de leurs activités. Une agente souligne :

« Le fait d'avoir un planning tout fait à l'intérieur de la trousse, c'est merveilleux. C'est un bon guide. Parce qu'habituellement, quand je monte une formation, ça me prend beaucoup plus de temps que ça. La recherche est faite, l'organisation... je trouve que c'est très, très bien préparé le manuel. »

Toutefois, les agentes ont mentionné que la profondeur du contenu et les nombreux sujets abordés par le programme (mauvais traitements physiques, psychologiques, syndrome du bébé secoué et actions préventives) font en sorte que beaucoup de temps est nécessaire pour lire, comprendre et intégrer l'ensemble des connaissances. 
Par ailleurs, le travail à deux a permis non seulement de renforcer le sentiment d'efficacité personnelle des agentes, mais aussi de profiter de la possibilité de répartir les tâches de préparation des activités de manière à ce que chaque agente se consacre aux tâches avec lesquelles elle se sent le plus à l'aise, diminuant d'autant le temps nécessaire pour effectuer ces tâches.

\section{Rôle des caractéristiques individuelles des agentes multiplicatrices.}

Le temps nécessaire à la préparation des activités de transfert n'est pas seulement lié au contenu du programme, mais aussi au sentiment d'efficacité personnelle des agentes multiplicatrices. La pression qu'elles ressentent face à l'importance de la thématique et leur volonté de transmettre le contenu du programme de la meilleure manière qui soit fait en sorte que le temps qu'elles souhaitent accorder à la préparation des activités est souvent au-delà du temps qu'elles peuvent réalistement consacrer à cette tâche. Ainsi, le temps nécessaire à la préparation est intimement lié aussi bien au besoin en formation que perçoivent les agentes qu'à leur sentiment d'efficacité personnelle puisque plus elles ont la perception que la formation Agir en milieu de garde répond à un besoin important et plus elles ont un faible sentiment d'efficacité personnelle, plus elles perçoivent que le temps nécessaire pour se préparer est grand.

\section{Rôle des caractéristiques organisationnelles.}

Par ailleurs lorsqu'il est question des influences sur le temps que les agentes peuvent accorder à la préparation et à l'animation d'activités de transfert, plusieurs considérations organisationnelles entrent en jeu. Une des raisons souvent mentionnées par les agentes multiplicatrices pour justifier le report d'activités de transfert est le manque ou la mobilité de personnel dans leur bureau coordonnateur. Le départ d'une collègue crée une charge de travail supplémentaire sur celles qui restent, obligeant ainsi la remise à plus tard des activités jugées moins pressantes. Ce genre d'évènement est d'autant plus problématique lorsque la collègue qui quitte est la partenaire de formation. En effet, lorsqu'une agente multiplicatrice se retrouve seule au sein de son bureau coordonnateur à assumer ce rôle, les difficultés et obstacles liés à la mise en œuvre d'une activité de transfert peuvent paraitre insurmontables.

Le temps nécessaire à l'organisation d'activités de transfert peut aussi être réduit lorsque les locaux et le matériel nécessaire à la présentation des activités sont disponibles au sein même du bureau coordonnateur. La présence de matériel et de locaux disponibles pour les activités à l'intérieur de l'organisation n'est par ailleurs qu'un élément du fonctionnement de l'organisation qui favorise la disponibilité des agentes pour la préparation d'activités. Ce fonctionnement organisationnel se reflète aussi dans la priorisation, dans le mandat de travail des agentes, de la tâche liée aux activités de transfert de connaissances aux responsables en service de garde. Pour certaines répondantes, préparer et animer ces activités est une part fondamentale de leur rôle au bureau coordonnateur alors que pour d'autres, leur mandat de travail est d'abord orienté vers le soutien individuel aux responsables. Cette différence au niveau des priorités organisationnelles fait en sorte que certaines agentes consacrent une plus grande part de leur temps aux activités de transfert 
alors que d'autres se consacrent plus largement aux tâches de soutien individuel. Le fonctionnement de l'organisation exerce aussi une influence via l'organisation du travail d'équipe. Dans certains bureaux coordonnateurs, les agentes ont des cases horaires réservées aux rencontres d'équipe, ce qui facilite grandement la préparation des activités en équipe de deux. Pour d'autres, le travail en équipe de deux, bien que permettant de diviser le travail, demeure complexe étant donné les horaires chargés de chacune et la nécessité de s'entendre sur la forme à donner aux activités.

\section{Rôle de la communauté.}

La participation, pour certaines agentes multiplicatrices, au projet Grandir ensemble offre un temps réservé où il est possible de discuter et de partager sur la préparation et l'animation d'activités de transfert, dont Agir en milieu de garde. Cela réduit d'autant le temps que les agentes doivent consacrer à ces tâches en d'autres moments.

Par ailleurs, le fait d'avoir de bons contacts avec certaines instances de la communauté, par exemple les écoles et les CSSS, facilite l'organisation matérielle des activités lorsque les locaux et le matériel (ordinateurs portables, projecteur, lecteur DVD, etc.) ne sont pas disponibles à l'intérieur même du bureau coordonnateur puisque ce sont souvent vers eux que les agentes se tournent pour emprunter le nécessaire. Le temps nécessaire à l'organisation des activités s'en trouve ainsi réduit.

\section{Résumé des influences et conséquences sur l'implantation.}

Le temps est un facteur central dans la décision d'implanter ou non le programme et dans le choix de la forme donnée aux activités. II influence le sentiment d'efficacité personnelle des agentes en leur permettant ou non de se préparer à leur convenance. Lorsque les agentes n'ont pas le temps qu'elles jugent nécessaire pour se préparer adéquatement, elles choisissent souvent d'orienter différemment leurs activités de transfert, soit en présentant seulement une partie du contenu du programme ou en optant pour une activité d'information qui demande moins de préparation puisqu'elles laissent une plus grande place aux interactions et réactions des responsables. Le facteur temps influence aussi la perception qu'elles ont des besoins des responsables puisque cette perception est directement liée à la nécessité de prioriser certaines thématiques de formation à défaut d'avoir le temps nécessaire pour présenter tout ce qui est jugé pertinent. La formation de deux agentes multiplicatrices par bureau coordonnateur est un moyen de réduire l'impact du manque de temps sur l'implantation. Toutefois, pour que cela puisse réellement avoir un effet bénéfique sur l'implantation, il faut que la culture organisationnelle du bureau coordonnateur facilite le travail d'équipe. Si tel n'est pas le cas ou si l'une des agentes multiplicatrices quitte l'organisation, les délais dans l'implantation engendrée par le manque de temps deviennent en soi un facteur défavorable à l'implantation. Ces délais font en sorte que le doute s'installe dans l'esprit des agentes multiplicatrices quant à leur capacité à jouer ce rôle au fur et à mesure qu'elles oublient les apprentissages faits lors de la formation du CLIPP. Ce doute s'installant, elles perçoivent le temps nécessaire à la préparation comme de plus en 
plus grand, alors que la cause initiale du délai est déjà un manque de temps. II va sans dire que ce cercle vicieux met en péril l'implantation de toutes les activités de transfert dans le cadre du programme.

\section{Discussion}

Les résultats de la présente étude permettent de mieux comprendre de quelle manière l'interaction entre les facteurs individuels, communautaires, organisationnels et propres au programme a influencé l'implantation du programme Agir en milieu de garde. En effet, l'évaluation de l'influence de ces facteurs sur l'implantation de ce programme démontre que les caractéristiques individuelles des agentes, les caractéristiques organisationnelles des bureaux coordonnateurs, les caractéristiques communautaires de ces bureaux ainsi que les caractéristiques du programme agissent ensemble autour de deux axes d'influence de niveau individuel (la perception des besoins et le sentiment d'auto-efficacité) et un axe organisationnel (le temps disponible).

Ainsi, lorsqu'il est question de ce qui a influencé l'adoption du programme Agir en milieu de garde, c'est-à-dire l'implantation ou non d'activités de transfert, les agentes multiplicatrices mentionnent presque uniquement des facteurs liés au manque de temps. Lorsque le manque de temps a entravé l'adoption du programme, cela s'explique par diverses situations, dont le manque ou la mobilité de personnel dans le bureau coordonnateur ou la priorisation dans la tâche des agentes du soutien personnel aux responsables en service de garde plutôt que de la préparation et de l'animation d'activités de transfert. Le temps disponible a non seulement influencé le choix des agentes d'adopter ou non le programme, mais aussi la manière dont celles-ci l'ont implanté. En effet, le temps disponible pour la préparation et l'animation des activités de transfert est, par rapport aux choix d'implantation, un facteur d'influence complémentaire au sentiment d'efficacité personnelle des agentes ainsi qu'à leur perception quant aux besoins des responsables en services de garde en matière de formation. Ce sont ces deux derniers facteurs qui ont principalement influencé les choix qu'ont faits les agentes quant au contenu et à la forme des activités de transfert qu'elles ont tenues, mais ces choix ont aussi dû être faits en considération du temps disponible pour mettre en place ces activités.

En ce qui concerne les besoins en formation des responsables en service de garde, les agentes multiplicatrices affirment que le programme Agir en milieu de garde répond effectivement à un besoin, et ce, à deux niveaux. II permet d'une part de faire prendre conscience aux responsables des situations de mauvais traitements que les enfants peuvent vivre dans leur milieu familial, des facteurs de risques liés à cette problématique, des signes à observer et des techniques à mettre en place pour prévenir et intervenir dans ces situations. D'autre part, le programme sensibilise les responsables en service de garde au fait qu'elles peuvent elles-mêmes être instigatrices de mauvais traitements et leur rappelle les gestes alternatifs, entre autres en leur rappelant que les agentes qui leur offrent les activités de transfert sont leurs alliées dans de telles situations. Ainsi, les agentes mentionnent l'importance d'allouer plus de temps, lors des activités de transferts, aux interactions entre les participantes et aux questions de ces dernières, en vu de 
mieux répondre aux besoins des responsables. Elles sont d'ailleurs relativement unanimes sur le fait que le programme répond à un besoin et traite d'un sujet incontournable pour leur milieu. À cet égard, les agentes ont exprimé leur volonté de mettre en place des activités de transfert dont la forme et le contenu attirent un maximum de participantes parmi leurs responsables en service de garde. Toutefois, les formules d'activités implantées pour ce faire varient largement d'une agente à l'autre entre autres en fonction de contraintes organisationnelles telles que l'autorisation aux agentes d'animer des activités de formation, mais aussi en fonction du sentiment d'efficacité personnelle de ces dernières.

Par ailleurs, parce qu'elles jugent le problème des mauvais traitement comme étant important, les agentes ont souhaité rejoindre le plus grand nombre possible de responsables grâce à leurs activités de transfert et elles ont aussi ressentie la nécessité de transmettre le contenu du programme de la meilleure façon qui soit. Ainsi, alors que la perception du besoin des responsables quant à ce problème a agi de manière favorable sur l'adoption du programme, il a nui au sentiment d'efficacité personnelle des agentes en leur imposant une pression concernant leur rôle d'agentes multiplicatrices. Cela dit, la formule « clé en main » du programme a fait en sorte que la perception de la crédibilité et de l'efficacité des agentes à jouer leur rôle soit rétablie, du moins en partie, autant au niveau personnel qu'organisationnel.

Alors que le temps disponible a d'abord influencé le choix des agentes d'adopter ou non le programme Agir en milieu de garde, il a aussi influencé la manière dont l'ont implanté celles qui l'ont adopté. En effet, bien que le besoin pour ce programme soit présent, ce ne sont pas toutes les agentes qui ont pu prendre le temps nécessaire à la préparation qui leur aurait permis d'avoir le sentiment d'efficacité personnelle nécessaire à la présentation des activités de transfert qu'elles souhaitaient mettre en place. Toutefois, le faible sentiment d'efficacité personnelle ne semble pas avoir amené les agentes à douter de la pertinence d'adopter le programme, mais plutôt les avoir amenées à repenser leurs formules d'activités de transfert, privilégiant souvent les activités d'informations au détriment des activités de formation. Les agentes dont le sentiment d'efficacité personnelle est moins fort ont donc souvent préféré jouer un rôle d'accompagnatrice informelle face au contenu du programme, et ce, en mettant l'accent sur des discussions et des échanges entre participantes plutôt que sur les présentations magistrales que le guide de formation préconise.

Les résultats de la présente étude montrent que, comme le soulignent Durlak et DuPre (2008), une implantation efficace dépend de l'interaction de plusieurs facteurs. Ces mêmes auteurs spécifient que ce qui est nécessaire à une implantation efficace est toutefois tributaire du contexte, bien que ces facteurs s'organisent généralement en quatre grandes catégories : les caractéristiques de l'innovation, les caractéristiques individuelles des acteurs de l'implantation, les caractéristiques organisationnelles du milieu d'implantation et finalement les caractéristiques communautaires de ce même milieu. Pour Agir en milieu de garde, ces quatre catégories de facteurs se sont déployées de manière à influencer l'implantation de trois grands enjeux : la perception qu'ont les agentes multiplicatrices des besoins en formation des responsables en services de garde, 
le sentiment d'efficacité personnelle des agentes face au programme et le temps disponible qu'ont ces dernières afin d'implanter le programme. Le fait que la perception des besoins en matière de formation des responsables en service de garde ait influencé l'implantation du programme est cohérent avec le propos de Hall et Hord (2006) qui suggère que la perception que l'innovation traite d'un sujet rejoignant les préoccupations des individus est liée à la décision d'adopter ou non l'innovation. Toutefois, Connell, Holmes, Voelkl et Bakalar (2002) ont démontré que des agents multiplicateurs peuvent être réticents à aborder certains sujets auprès de leurs participants lorsqu'il s'agit de thèmes complexes ou jugés sensibles, et cela, au-delà de leur perception des besoins des participants quant à ces thèmes. C'est aussi ce qu'expriment les agentes multiplicatrices du programme Agir en milieu de garde lorsqu'elles mentionnent que leur sentiment d'efficacité personnelle est affecté par le fait que la thématique des mauvais traitements chez les jeunes enfants est jugée sensible et qu'ainsi elles ont parfois préféré jouer un rôle plus informel dans la présentation de ces thématiques en privilégiant des activités d'information. Toutefois, les agentes du programme Agir en milieu de garde comme ceux du programme de transfert de connaissances par agents multiplicateurs sur les soins à la démence évalué par Connell et al.(2002), ont en bon nombre préféré trouver des manières alternatives de présenter le contenu lorsqu'elles se sentaient peu efficaces plutôt que de ne pas l'implanter, et cela parce qu'elles jugeaient que le contenu répondait à un besoin. Connell et al. (2002) mentionnent aussi que la difficulté de trouver suffisamment de temps pour préparer des activités de formation influence l'implantation d'un programme par agents multiplicateurs, comme c'est le cas dans la présente étude.

\section{Limites}

Les résultats de la présente étude doivent être interprétés en prenant en considération un certain nombre de limites. D'abord, les entretiens avec les agentes multiplicatrices ont été effectués par l'équipe d'évaluation du CLIPP. Puisque les interviewers se sont présentés comme tels aux participantes, cela a pu engendrer un biais de désirabilité sociale chez celles-ci et influencer leurs réponses. Le choix méthodologique de procéder aux entrevues par téléphone peut aussi avoir engendré une certaine perte d'information puisque le langage non verbal des participants n'a pu être pris en considération. Toutefois, ce choix se justifie par la volonté d'inclure les agentes multiplicatrices de toutes les régions du Québec dans cette étude dont les ressources étaient limitées.

Par ailleurs, les entretiens ont été analysés après que les résultats du volet précédent de l'étude ont été connus. Cela a pu orienter le processus d'analyse du présent volet en favorisant l'élaboration de conclusions complémentaires à celles du premier volet. Cela dit, un seul des chercheurs parmi les trois membres de l'équipe d'analyse était au fait des résultats du premier volet de l'étude lors des analyses du second volet. Ainsi, la mise en place d'un dispositif d'accord interjuges entre trois chercheurs lors des procédures de codage et d'analyse permet de garantir une certaine validité interne aux résultats de cette étude.

Toutefois, il est difficile, voire impossible, de qualifier la validité externe de cette étude. Cette dernière vise à documenter les processus d'influence sur 
l'implantation d'un programme spécifique, le programme Agir en milieu de garde, et non à conclure sur les processus d'influence des initiatives de transfert de connaissances en général. La généralisation de ses résultats à d'autres programmes ou contextes doit être faite avec prudence.

\section{Conclusion}

Les programmes par agents multiplicateurs sont un moyen efficient de transférer des connaissances à un large public (Culp et al., 2003). Toutefois, la fidélité du transfert dans le cadre de ces programmes peut-être affectée par le fait que les agents multiplicateurs sont moins enclins à présenter les éléments plus novateurs (Hahn et al., 2002) ou ceux jugés complexes ou sensibles (Connell et al., 2002). Des instructions claires et précises incluses dans un manuel d'implantation permettent d'augmenter la fidélité d'implantation (Luborsky \& DeRubeis, 1984). Les instructions se trouvant dans le guide du programme Agir en milieu de garde, sous forme de suggestions ou de recommandations plutôt que de consignes ont ainsi probablement contribué à laisser une grande place à l'influence du contexte, via les trois grands axes présentés précédemment. Ainsi, il est probable que les instructions souples du guide ont favorisé l'implantation d'activités aux contenus et aux formats choisis par les agentes pour mieux les adapter à leur contexte. Aurait-il été préférable d'imposer aux agentes multiplicatrices des barèmes d'implantation plus stricts au nom de la fidélité d'implantation? II n'y a pas de consensus actuellement en sciences sociales à savoir si des exigences rigides en ce qui concerne la fidélité d'implantation sont ou non souhaitables (Dusenbury, Brannigan, Falco et Hansen, 2003). Berman et McLaughlin (1976) soulignent que la flexibilité offerte aux acteurs dans l'implantation augmente les probabilités qu'a un programme d'être implanté. En ce qui concerne Agir en milieu de garde, il peut en effet être postulé que si des exigences plus spécifiques avaient été émises dans le guide de formation et lors des formations offertes par le CLIPP, les agentes multiplicatrices jugeant ces instructions peu appropriées à leur milieu ou ne se sentant pas à l'aise par rapport à celles-ci se seraient abstenues d'implanter le programme. II est donc possiblement préférable que la souplesse des recommandations d'implantation du guide ait permis à ces agentes d'adapter le format et le contenu de leurs activités de transfert à leur milieu et à leur niveau de sentiment d'efficacité personnelle.

Cela dit, comme le soulignent Ridgely et Jerrell (1996), il est approprié de se questionner sur les modifications faites à un programme au fil de son implantation : ces modifications sont-elles volontaires, nécessaires et appropriées dans un contexte donné ou sont-elles des erreurs? À supposer que les deux éléments (décisions et erreurs d'implantation) aient une part à jouer dans la fidélité d'implantation, un monitorage de l'implantation et un complément de formation auprès des acteurs de l'implantation pourraient être appropriés. Ces deux stratégies ont été démontrées efficaces pour augmenter la fidélité d'implantation (DuFrene, Noell, Gilbertson \& Duhon, 2005; Greenwood, Tapia, Abbott \& Walton, 2003).

À la suite de l'évaluation de l'implantation et des facteurs d'influence du programme Agir en milieu de garde, il est suggéré qu'offrir un complément de formation aux agentes multiplicatrices pourrait être un moyen d'augmenter la fidélité d'implantation. Cela permettrait de tenter de diminuer les modifications faites au 
programme par erreur le cas échéant, tout en laissant aux agentes multiplicatrices la latitude d'implantation leur permettant d'adapter celui-ci à leur contexte propre. Ce complément de formation permettrait aussi aux agentes qui n'ont pu implanter le programme à ce jour de rafraichir leurs connaissances, diminuant ainsi l'impression qu'elles ont qu'il est d'autant plus ardu de préparer des activités si longtemps après la formation initiale et augmentant ainsi la possibilité qu'elles adoptent le programme.

\section{Références}

Altschuld, J. W., Kumar, D. D., Smith, D. W., \& Goodway, J. D. (1999). Schoolbased educational innovations: Case illustrations of context sensitive evaluations. Family and Community Health, 22, 66-79.

Berman, P., \& McLaughlin, M. W. (1976) Implementation ofeducationalinnovation. The Educational Forum, 40, 345-370. doi: $10.1080 / 00131727609336469$

Briand-Lamarche, M. \& Dagenais, C. (2012). Évaluation des processus d'implantation d'un programme de transfert de connaissances par agents multiplicateurs pour la prévention des mauvais traitements. Revue de Psychoéducation, 41 (1), pages à venir.

Bronfenbrenner, U. (1977). Toward an experimental ecology of human development. American Psychologist, 32, 513-531.

Centre de liaison sur l'intervention et la prévention psychosociales (2004). Projet de prévention : mauvais traitements physiques et enfants secoué. Étude des besoins de formation du personnel des CPE et des policiers. Montréal : Centre de liaison sur l'intervention et la prévention psychosociale.

Centre de liaison sur l'intervention et la prévention psychosociales (2006). Trousse de formation à la prévention des mauvais traitements physiques et psychologiques envers les jeunes enfants : Agir en milieu de garde. Rapport final d'évaluation. Montréal : Centre de liaison sur l'intervention et la prévention psychosociale.
Centre de liaison sur l'intervention et la prévention psychosociales (2008). Rapport d'évaluation des réactions sur la formation à la prévention des mauvais traitements physiques et psychologiques envers les jeunes enfants Agir en milieu de garde. Montréal : Centre de liaison sur l'intervention et la prévention psychosociale.

Connell, C. M., Holmes, S. B., Voelkl, J. E., \& Bakalar, H. R. (2002). Providing dementia outreach education to rural communities: Lessons learned from a train-the-trainer program. Journal of Applied Gerontology, 21 (3), 294-313. doi:10.1177/073346480202100302

Culp, K. M., Gersick, A., Martin, W., Nudell, H., Pederson, S., \& Shankar, S. (2003). Selected Findings from the Evaluation of Intel Teach to the Future: The Center of Children and Technology.

Dane, A. V., \& Schneider, B. H. (1998). Program integrity in primary and early secondary prevention: Are implementation effects out of control. Clinical Psychology Review, 18, 23-45. doi: 10.1016/S0272-7358(97)00043-3

Delaronde, S., King, G., Bendel, R., \& Reece, R. (2000). Opinions among mandated reporters toward child maltreatment reporting policies. Child Abuse \& Neglect, 24 (7), 901-910. doi: 10.1016/ S0145-2134(00)00151-4

Dufrene, B. A., Noell, G. H., Gilbertson, D. N., \& Duhon, G. J. (2005). Monitoring implementation of reciprocal peer tutoring: Identifying and intervening with students who do not maintain accurate implementation. School Psychology Review, 34, 74-86. 
Durlak, J., \& DuPre, E. (2008). Implementation matters: A review of research on the influence of implementation on program outcomes and the factors affecting implementation. American Journal of Community Psychology, 41 (3), 327350. doi:10.1007/s10464-008-9165-0

Dusenbury, L., Brannigan, R., Falco, M., \& Hansen, W. B. (2003). A review of research on fidelity of implementation: Implications for drug abuse prevention in school settings. Health Educ. Res., 18 (2), 237-256.

Fernandes, S., Kamienski, C., Kelner, J., Mariz, D., \& Sadok, D. (2008). A stratified traffic sampling methodology for seeing the big picture. Computer Networks, 52, 2677-2689. doi : 10.1016/j. comnet.2008.05.011

Fondation Lucie, \& André Chagnon (2009). Grandir, s'unir et agir pour enrichir nos pratiques. Récupéré de : http://www. fondationchagnon.org/fr/PDF/2009 colloque_grandir_s_unir_agir.pdf

Fraser, M. W. (2004). Intervention research in social work: Recent advances and continuing challenges. Research on Social Work Practice, 14, 210-222. doi : $10.1177 / 1049731503262150$

Gaston, V., \& Sutherland, J. (1999). Bibliographie sélective et annotée sur le signalement par des professionnels de l'éducation de la maltraitance envers les enfants. Ottawa, ON : Santé Canada - Division de la violence envers les enfants, Direction générale de la protection de la santé.

Gira, E. C., Kessler, M. L., \& Poertner, J. (2004). Influencing social workers to use research evidence in practice: Lessons from medicine and the allied health professions. Research on Social Work Practice, 14, 68-79. doi : 10.1177/1049731503262128

Goodman, R., Speers, M., McLeroy, K., Fawcett, S., Kegler, M., Parker, E., \& Wallerstein, N. (1998). Identifying and defining the dimensions of community capacity to provide a basis for measurement. Health Education Behavior, 25, 258-278. doi : $10.1177 / 109019819802500303$
Greenhalgh, T., Robert, G., Macfarlane, F., Bate, P., \& Kyriakidou, O. (2004). Diffusion of innovations in service organizations: Systematic review and recommendations. Milbank Quarterly, 82 (4), 581-629. doi: 10.1111/j.0887$378 X .2004 .00325$

Greenwood, C. R., Tapia, Y., Abbott, M., \& Walton, C. (2003). A buildingbased case study of evidence-based literacy practices: Implementation, reading behavior, and growth in reading fluency, K-4. The Journal of Special Education, 37, 95-110. doi : 10.1177/00224669030370020401

Hahn E.J., Noland, M.P., Rayens, M.K., \& Christie, D.M. (2002). Efficacy of training and fidelity of implementation of the life skills training program. Journal of school health, 72 (7), 282-287. doi: 10.1111/ j.1746-1561.2002.tb01333.x

Hall, G. E., \& Hord, S. M. (2006). Implementing change: Patterns, principles, and potholes. Needham Heights, MA: Allyn and Bacon.

Hinds P.J., Patterson M., \& Pfeffer J, (2001). Bothered by abstraction: the effect of expertise on knowledge transfer and subsequent novice performance. Journal of Applied Psychology; 86, 1232-1243. doi : 10.1037/0021-9010.86.6.1232

Howard, M. O., McMillen, C. J., \& Pollio, D. E. (2003). Teaching evidence-based practice: Toward a new paradigm for social work education. Research on Social Work Practice, 13, 234-259. doi : 10.1177/1049731502250404

Lee, S., Altschul, I., \& Mowbray, C. (2008). Using planned adaptation to implement evidence-based programs with new populations. American Journal of Community Psychology, 41 (3), 290303. doi: 10.1007/s10464-008-9160-5

Lessard-Hébert, M., Goyette G., \& Boutin, G. (1996). La recherche qualitative. ( $2^{\mathrm{e}}$ éd.). Québec : Éditions nouvelles.

Levy, K., Hirsch, E., Aghababian, R.V, Segall, A., \& Vanderschmidt, H. (1999). Radiation accident preparedness: Report of a training program involving the United States, Eastern Europe, 
and the Newly Independent States. American Journal of Public Health. 89 (7), 1115-1117.

Luborsky, L., \& DeRubeis, R. J. (1984) The use of psychotherapy treatment manuals: A small revolution in psychotherapy research style. Clinical Psychology Review, 4, 5-14. doi: 10.1016/0272-7358(84)90034-5

McClelland, J.W., Irving, L.M., Mitchell, R.E., Bearon, L.B., \& Webber, K.H. (2002). Extending the reach of nutrition education for older adults: Feasibility of a train-the-trainer approach in congregate nutrition sites. Journal of Nutrition Education and Behavior, 34, S48- S52. doi: 10.1016/S1499-4046(06)60311-4

Miles, M. B., \& Huberman, A. M. (2e éd.). (2003). Analyse des données qualitatives. Bruxelles : De Boeck \& Larcier.

Miller, R. L., \& Shinn, M. (2005). Learning from communities: Overcoming difficulties in dissemination of prevention and promotion efforts. American Journal of Community Psychology, 35 (3/4), 143-157. doi : 10.1007/s10464-0053395-1

Orfaly, R. A., Frances, J. C., Campbell, P., Whittemore, B., Joly, B., \& Koh, H. (2005). Train-the-trainer as an educational model in public health preparedness. Journal of Public Health Management \& Practice, 11, 123-127.

Patton, M. Q. (2e éd.). (1990). Qualitative evaluation and research method. California: Newbury Park. New York: Sage Publications.

Poupart, J., Deslauriers, J.P., Groulx, L.H., Laperrière, A., Mayer, R., \& Pires, A.P. (1997). La recherche qualitative: Enjeux épistémologiques et méthodologiques. Boucherville : Gaëtan Morin Éditions.

Ridgely, M. S., \& Jerrell, J. M. (1996) Analysis of three interventions for substance abuse treatment of severely mentally ill people. Community Mental Health Journal, 32, 561-572. doi: 10.1007/ BF02251066
Riley, B. L., Taylor, S. M., \& Elliott, S. J. (2001). Determinants of implementing heart healthy promotion activities in Ontario public health units: A social ecological perspective. Health Education Research, 16, 425-441.

Ringwalt, C., Ennet, S., Vincus, A., Thorne, J., Rohrbach, L. A., \& SimonsRudolph, A. (2002). The prevalence of effective substance use prevention curricula in U.S. middle schools. Prevention Science, 3 (4), 257-265. doi :10.1023/A:1020872424136

Schorr, L. B. (2003). Determining "what works" in social programs and social policies: Toward a more inclusive knowledge base. Washington, DC: Brookings Institution.

Shediac-Rizkallah, M. C., \& Bone, L. R. (1998). Planning for the sustainability of community-based health programs: Conceptual frameworks and future directions for research, practice and policy. Health Education Research, 13, 87-108.

Strauss, A. \& Corbin, J. (1990). Basics of Qualitative Research : Grounded Theory Procedures and Techniques. Newbury Park. Sage Publications.

Tashakkori, A., \& Teddlie, C., (2003). Handbook of mixed methods in social \& behevioral research. California: Thousand Oaks.

Tourigny, M., Mayer, M., Wright, J., Lavergne, C., Trocmé, N., Hélie, S. \& Larrivée, M-C. (2002). Étude sur l'incidence et les caractéristiques des situations d'abus, de négligence, d'abandon et de troubles de comportement sérieux signalées à la Direction de la protection de la jeunesse au Québec (ÉlQ). Montréal : Centre de liaison sur l'intervention et la prévention psychosociales (CLIPP).

Trocmé, N., McPhee, D., Tam, K. K., \& Hay, T. (1994). Ontario incidence study of reported child abuse and neglect. Toronto, ON: Institute for the Prevention of Child Abuse.

Van der Maren, J.M. (2e éd.). (1996). Méthodes de recherche pour l'éducation. Bruxelles: De Boeck-Université. 
Wandersman, A. (2003). Community Wandersman, A., \& Florin, P. (2003). science: Bridging the gap between science and practice with communitycentered models. American Journal of Community Psychology, 31, 227-242. doi : 10.1023/A:1023954503247

Community interventions and effective prevention. American Psychologist, 58, 441-448. doi: 10.1037/0003-066X.58.67.441

Wandersman, A., Duffy, J., Flaspohler, P., Noonan, R., Lubell, K., Stillman, L. \& Saul, J. (2008). Bridging the gap between prevention research and practice: The interactive systems framework for dissemination and implementation. American Journal of Community Psychology, 41 (3), 171181. doi: 10.1007/s10464-008-9174-z 